Cahiers de recherches médiévales

\title{
Le Iulius Coesar de Marc-Antoine de Muret
}

\section{Virginie Leroux}

\section{(2) OpenEdition}

Journals

Édition électronique

URL : https://journals.openedition.org/crm/2576

DOI : $10.4000 / \mathrm{crm} .2576$

ISSN : 1955-2424

Éditeur

Honoré Champion

Édition imprimée

Date de publication : 30 juin 2007

Pagination : 207-223

ISSN : 1272-9752

\section{Référence électronique}

Virginie Leroux, "Le lulius Cæsar de Marc-Antoine de Muret », Cahiers de recherches médiévales [En ligne], 14 spécial | 2007, mis en ligne le 30 juin 2010, consulté le 15 décembre 2022. URL : http:// journals.openedition.org/crm/2576; DOI : https://doi.org/10.4000/crm.2576 


\section{RM}

\section{Le Iulius Casar de Marc-Antoine de Muret}

Marc-Antoine de Muret semble être le premier à avoir eu l'idée de mettre en scène la mort de César, innovation qui suscitera de nombreux émules. Son Iulius Caesar figure en tête du recueil des Iиuenilia, paru à Paris en 1552, à un moment où le jeune Muret, récemment arrivé dans la capitale, veut consolider sa position dans la République des Lettres, mais la tragédie a été composée quelques années auparavant $^{1}$. Elle l'était assurément en $1549^{2}$ et les témoignages de Joseph Scaliger et de Montaigne - malheureusement sujets à caution - fournissent la date de 1544. Le premier soutient que Muret a composé sa tragédie à Auch lorsqu'il avait 18 ans et Montaigne affirme avoir interprété à Bordeaux une tragédie de Muret, alors qu'il avait à peine onze ans ${ }^{3}$. Sans entrer ici dans le détail des nombreuses datations proposées par les savants, on peut retenir que le Iulius Caesar a, selon toute probabilité, été composé pour être représenté dans un collège, celui d'Auch ou le collège de

${ }^{1}$ M. A. Mureti Iuuenilia, Parisiis, ex officina Viduæ Mauricii a Porta in clauso Brunello, ad D. Claudii insigne, 1552. En 1995 ont vu le jour une édition critique de l'ensemble du recueil, procurée par D. Schmitz (Frankfurt am Main, Peter Lang) et une édition du Iulius Caesar procurée par P. Blanchard (Thonon-les-Bains, Alidades). La version remaniée de ma thèse de Doctorat, consacrée au recueil, soutenue en 1999, à l'Université de Paris III, sous la direction de R. Martin, doit paraître à Genève, chez Droz en 2007. La biographie déjà ancienne de Ch. Dejob, Marc-Antoine Muret: un professeur français dans la seconde moitié du XVI siècle, Paris, 1881 (réimpr. Genève, 1970) et le mémoire d'habilitation de J.-E. Girot, Un humaniste français à Rome à la fin de la Renaissance : Marc-Antoine Muret et les belles-lettres, soutenu en 2003, à l'Université de Paris III, sous la direction de M. Magnien, traitent surtout de la période italienne, on les complètera par l'article de R. Trinquet, «Recherches chronologiques sur la jeunesse de M.A. Muret», B.H.R., 27, 1965, p. 272-85. Sur le Iulius Caesar et sa postérité on consultera en particulier les références suivantes: J. Blänsdorf, «Die Verwandlung der senecanischen Trägodie in Marc-Antoine Murets 'Julius Cæsar' und Jacques Grevins 'César' », International Journal of the Classical Tradition, 1, 1994, 2, p. 5874 ; E. Dutertre, «À propos de quelques tragédies de la mort de César des XVI ${ }^{\mathrm{e}}$ et XVII ${ }^{\mathrm{e}}$ siècles », Littératures classiques, 16, 1992, p. 199-227 ; E. Ginsberg, «The Legacy of MarcAntoine Muret's 'Julius Cæsar' », ACNL Lovaniensis, éd. J. Ijsewijn et E. Kessler, LeuvenMünchen, 1973, p. 247-52; H. Lamarque, «La première tragédie 'prétexte' de la Renaissance: le Julius Casar de Marc-Antoine Muret», Rome et le tragique, éd. M.-H. Garelli-François, Pallas, 49, 1998, p. 247-65 ; M. Piccolomini, The Brutus Revival. Parricide and Tyrannicide during the Renaissance, Carbondale, Southern Illinois University, 1991; J. Pineaux, "César dans la tragédie humaniste de la Renaissance française », Présence de César, éd. R. Chevallier, Paris, Cæsarodunum 20bis, 1985, p. 213-22.

${ }^{2}$ D'après un passage des Leçons Variées et une lettre au cardinal de Tournon (Opera omnia, éd. H. Frotscher, 1834, t. II, p. 85 et t. III, p. 65), R. Trinquet déduit que Muret a envoyé en 1549 un exemplaire de la tragédie au cardinal (art. cit., p. 273-74).

${ }^{3}$ J.-J. Scaliger, Fabula Burdonice Confutatio, Lugduni Batavorum, Ex officina Ioannis Pratii, 1608, p. 387 et Montaigne, Essais, I, 26, éd. Villey, t. I, p. 176B.

Cahiers de Recherches Médiévales, 14spé, 2007 
Guyenne dont les programmes incluaient des représentations dramatiques ${ }^{4}$. De fait, la tragédie constitue le meilleur entraînement à l'art oratoire et fournit des exemples édifiants aux jeunes élèves. Cette mission morale du genre, rappelée par Muret dans son commentaire du De Officiiis ${ }^{5}$, est soulignée par les théoriciens de la Renaissance. Francisco Robortello recourt pour définir le genre aux catégories épidictiques de l'éloge et du blâme ${ }^{6}$ et Jules-César Scaliger prescrit aux auteurs de tragédie, «de veiller à ce que les spectateurs soient, non tant étonnés et ébranlés comme chez Eschyle, qu'instruits, émus et charmés ${ }^{7}$. Ce dernier témoignage a d'autant plus d'importance que le Julius Casar est probablement le fruit de discussions de Muret avec Scaliger et que le vieux maître, particulièrement fier de son prénom et prompt à s'identifier au dictateur, a peut-être joué un rôle dans le choix du sujet de la pièce. Sans aller jusqu'à lire comme le fait Pierre Blanchard dans le meurtre de César une illustration du concept freudien de meurtre du père, il est indéniable que la pièce constitue un hommage 8 .

Lors de sa parution en 1552, la tragédie doit remplir une double mission : répondre aux attentes de Du Bellay à un moment où la réflexion théorique des Français sur le genre était encore peu développée et fournir un modèle aux poètes de la nouvelle école ou, selon l'objectif formulé dans la préface des Iuиenilia, un équivalent latin de leurs œuvres en langue vernaculaire'. Proche de Jodelle, dont deux dédicaces figurent dans l'appareil liminaire des Iunenilia, Muret n'ignore pas que ce dernier est précisément en train de préparer sa Cléopâtre et son Eugène qui seront représentés en janvier-février $1553^{10}$.

À travers quelques exemples, nous étudierons comment Muret concilie les enjeux philologiques et éthiques en inscrivant les données fournies par les historiens antiques dans une triple tradition, dramatique, rhétorique et philosophique. Le Iulius Cassar fait écho aux réflexions de l'époque concernant le pouvoir, les devoirs de

\footnotetext{
${ }^{4}$ Voir E. Gaullieur, Histoire du collège de Guyenne d'après un grand nombre de documents inédits, Sandoz et Fischbacher, 1874, p. 253.

${ }^{5}$ In hunc usum comparate sunt Comœdice et Tragodice, quibus spectandis vulgarium animi vitiorum deformitatem, et virtutum pulchritudinem quoquo modo videre possent. Ad De Officiis, 1, 3 (1574), M. Antonii Mureti (...) notce in tres libros de Officiis et in quinque de Finibus, in Orationem Ciceronis pro Deiotario (...), Ingolstadii, A. Sartorius, 1602, p. 225.

${ }^{6}$ Si recitatio atque imitatio uirtutum fit et laudum praclari alicuius uiri, incitantur homines ad uirtutem: si rursus uitia reprasentantur, ab his homines multum deterrentur, maioreque quadam ui repelluntur, quam si alia quauis hortatione utaris. In librum Aristotelis De arte Potica explicationes (...), Florentiæ, in officina L. Torrentini, 1548, p. 3.

${ }^{7}$ Neque enim eo tantum spectandum est, ut spectatores uel admirantur uel percellantur : id quod Aschylum factitasse aiunt Critici: sed et docendi et mouendi, et delectandi. Pœtices libri septem, Lyon, A. Vicentium, 1561, 3, 97, p. 145.

${ }^{8}$ Introduction de l'édition citée, p. 24-28.

${ }^{9}$ Iunenilia, 1552, éd. cit., p. 9-10.

${ }^{10}$ Voir E. Buron, Dessous un silence obstiné : histoire des xuvres et de la théorie poétique d'Étienne Jodelle, Thèse de Doctorat, sous la direction de M. Simonin, Tours, 1997, p. 15361 (gestation de Cléopâtre Captive) et p. 86-120 (la restauration du théâtre antique, janvierfévrier 1553).
} 
l'homme d'état ou l'assassinat politique, mais aussi aux débats théologiques contemporains.

\section{L'inscription de la mort de César dans un schéma tragique}

On a souvent critiqué le caractère squelettique de la tragédie de Muret, trois fois plus courte que celles de Sénèque ou de son contemporain Buchanan ${ }^{11}$. Cette brièveté est, en fait, le fruit d'une observation scrupuleuse des règles d'unité d'action et de temps que les hommes de la Renaissance formulèrent à partir d'Aristote ${ }^{12}$. Muret circonscrit l'action aux Ides de mars - alors que Grévin intègre la vengeance de Marc-Antoine et que Shakespeare mène l'action jusqu'au suicide de Brutus - et fait quasiment énoncer à Brutus la convention de l'unité de temps lorsqu'il évoque au début de l'acte II le lever et le coucher du soleil (v. 147-48). La comparaison avec les tragédies de ses successeurs souligne la différence des esthétiques. Muret a très clairement fait le choix de la concentration. Il a donc volontairement laissé de côté de nombreux détails que les historiens lui fournissaient et exclu tout pittoresque extérieur. Par exemple, il ne dit rien du rhéteur Artémidore de Cnide qui, selon Plutarque $^{13}$, tenta de révéler le complot mais en fut empêché par la foule, alors que l'épisode sera exploité par Shakespeare (Iulius Caesar III, 3 et III, 1) et par Scudéry (La mort de César, III, 6 et IV, 6). Cette économie de détail est subordonnée à une esthétique de la clarté et de l'épure et contribue à l'efficacité de la pièce en soulignant l'accélération du destin.

Dès l'Antiquité, les historiens inscrivirent la mort de César dans un schéma tragique et insistèrent sur le rôle de la Fortune ${ }^{14}$. C'est ainsi qu'ils mentionnent les nombreux présages qui précédèrent la mort du dictateur et notamment le songe prémonitoire de sa femme Calpurnie et la tentative de celle-ci pour dissuader son mari de se rendre au sénat, tentative que l'intervention de Brutus voua à l'échec. Muret met à profit la dramatisation déjà opérée par ses sources antiques et indique la valeur topique des éléments qu'il sélectionne. Il intègre ainsi l'épisode du songe de Calpurnie au troisième acte de la tragédie dans un dialogue type entre l'héroïne et sa nourrice (v. 240-305), et signale que le motif est un «lieu» du genre tragique en imitant notamment l'Octavie du pseudo-Sénèque et le récit du songe de Storgè dans la Jeph-

\footnotetext{
${ }^{11}$ Le Iulius Casar ne comprend que 570 vers, alors que les tragédies de Buchanan en comptent respectivement 1360 pour Baptistes et 1450 pour Jephtes, ce qui correspond à peu près à la norme des tragédies de Sénèque (si on excepte les Phéniciennes et leurs 665 vers).

${ }^{12}$ Si la Poétique d'Aristote formule explicitement le principe de l'unité d'action (1450b-51b), l'unité de temps n'est qu'évoquée au cours d'une comparaison entre épopée et tragédie : alors que l'épopée ne connaît pas de limitation dans le temps, la tragédie «essaie autant que possible de se dérouler durant une seule révolution du soleil ou de ne guère s'en écarter» (1449b, trad. M. Magnien, Paris, Le livre de poche, classique, p. 92). Sur les interprétations de ce passage par les théoriciens de la Renaissance, voir M. T. Herrick, The Fusion of Horatian and Aristotelian Literary Criticism, 1531-1555, The University of Illinois Press, 1946, p. 7984.

${ }^{13}$ Vies parallèles. César 65.

${ }^{14}$ Nicolas de Damas, Vie de César, 23, 81-84 ; Suétone, Vie des Douze Césars. Jules César, 81 ; Plutarque, César, 63-66 ; Appien, Guerre civile, 2, 16, 116 et 2, 21, 152 ; Dion Cassius, Histoire romaine, 44, 17-18.
} 
tes de Buchanan ${ }^{15}$. Des deux versions antiques, Muret choisit la plus effrayante, celle qui offre la vision la plus saisissante, César baignant dans son sang, le torse percé de nombreux coups :

\author{
CAL. Casar meus, nutrix mea, heu, Caesar meus, \\ Meus ille Caesar, quo mea innixa est salus, \\ Mihi visus vlnas inter effusus meas, \\ Iacêre multo sanguine, et tabo fluens, \\ Multísque plagis pulcra fossus pectora. (v. 265-75) \\ Mon César, chère nourrice, hélas, mon César, \\ le grand César dont dépend mon salut \\ m'est apparu : reposant dans mes bras, \\ il gisait dans le sang, ses blessures coulaient \\ et son beau torse était percé de nombreux coups ${ }^{16}$.
}

Suscitant crainte et pitié, le passage répond à la finalité assignée par Aristote à la tragédie et annonce l'évocation du meurtre par Cassius à l'acte V - car conformément aux recommandations d'Horace, le meurtre ne sera pas représenté sur scène mais « confié à l'éloquence d'un témoin ${ }^{17}$.

La tentative de Calpurnie pour empêcher César de se rendre au sénat et l'intervention de Decimus Brutus fournissent de même une scène type des tragédies de Sénèque, que Florence Dupont appelle la scène de duel ${ }^{18}$. Alors que Suétone mentionne rapidement l'hésitation de César (Jules César, 81), Muret dramatise la notation de l'historien en utilisant le cliché du navire ballotté par la tempête, fréquente dans les tragédies de Sénèque pour exprimer les tourments d'une âme incertaine ou encore les vicissitudes de l'existence humaine ${ }^{19}$ :

CAES. Incertus animi, et huc, et illuc distrahor:

Qualis per aequor concitum bacchantibus

Deprensa ventis fertur incerto ratis

Agitata cursu : pellit illinc Africus

\footnotetext{
${ }^{15}$ Sénèque, Octavie, v. 712-18 et 734. Les emprunts à Buchanan sont signalés par R. Lebègue, La tragédie religieuse en France. Les débuts (1514-1573), Paris, Champion, 1929, p. 246.

${ }^{16}$ Muret suit la version présentée par Valère-Maxime $(1,7,2)$ et Plutarque (César, 63, qui rapporte les deux). Selon Suétone (Jules César, 81) et Dion Cassius (Histoire romaine, 44, 17, 1), Calpurnie aurait vu le faite de sa maison s'écrouler. La mention des nombreuses blessures rappelle les récits antiques de la mort du dictateur qui insistent sur le grand nombre de coups de poignards reçus par César, 23 selon Suétone (Jules César, 82) ou Plutarque (César, 66, 14) et même 35 selon Nicolas de Damas (Vie de César, 24).

${ }^{17}$ Horace, Art poétique, v. 182-188.

${ }^{18}$ Le Théâtre latin, Paris, Armand Colin, Cursus, 1988, p. 72.

${ }^{19}$ Voir notamment Agamemnon, v. 138-44 ou Thyeste, v. 438-39. Sur les autres occurrences de cette métaphore chez Sénèque, voir A. Cattin, Les thèmes lyriques dans les tragédies de Sénèque, Neuchâtel, Delachaux et Niestlé, 1963, p. 80-83, et M. Armisen-Marchetti, Sapientia facies. Etude sur les images de Sénèque, Paris, Les Belles Lettres, 1989, p. 142-43.
} 
Creber procellis, Eurus hinc, illinc Notus :

Sic me hinc tua, Calpurnia, inflectunt preces,

Hinc dicta Bruti : sed tamen quando semel

Vel cadere prastat, quàm metu longo premi :

Non si trecentis vocibus vatum auocer,

Non si ipse voce propria prasens deus

Moneat pericli, atque hîc manendum suadeat,

Me continebo. Desine, vxor, conqueri.

Eamus : omnis iacta nobis alea est. (Iulius Casar, v. 379-91)

CES. L'âme incertaine, ballotté de-ci de-là, je suis comme l'esquif sur la mer en furie, emporté par les vents menant leur bacchanale ${ }^{20}$ en une course incertaine : poussé tantôt par l'Africus, fertile en orages, de ça par l'Eurus, de là par le Notus. Ainsi, Calpurnie, tes prières m'entraînent d'un côté, les paroles de Brutus d'un autre. Mais puisqu'il vaut mieux tomber une bonne fois qu'être rongé par une longue angoisse ; non, même si j'en étais détourné par trois cents devins, non, même si un dieu en personne de sa propre voix m'avertissait du danger et m'enjoignait de demeurer ici, je ne resterai pas. Cesse de gémir, ma femme.

Allons : le sort en est jeté.

En reprenant avec humour la formule célèbre qui accompagne le passage du Rubicon, Muret associe la décision de César au topos de la Fortuna Caesaris, mais cette fois l'issue sera fatale. C'est pourquoi le chœur qui suit la scène, évoquant les vaines prophéties de Cassandre, souligne l'ironie tragique de la décision du dictateur.

De fait, ce sont surtout les chœurs qui inscrivent le meurtre du dictateur dans un schéma tragique. Le premier chœur caractérise l'action par un topos du genre, l'instabilité de la fortune qui frappe les puissants. Cette conception abstraite de la Tychè est relayée dans le chœur III par la figure de Némésis, figure éthique qui châtie les orgueilleux et les puissants. César y apparaît comme un héros abattu par la Fortune au faîte de sa gloire. Le motif était déjà présent au chant VII de la Pharsale, lorsque Lucain apostrophe Brutus au cœur de la bataille et lui indique qu'il perd sa peine puisque César «n'a pas encore atteint le sommet du pouvoir, dépassé ce faîte de la grandeur humaine, d'où l'on opprime tout, pour mériter des destins une mort si fameuse » (Nondum attigit arcem,/ iuris et humani columen, quo cuncta premuntur,/ egressus meruit fatis tam nobile letum. $)^{21}$. Dans le deuxième chœur, Muret accentue l'adaptation générique en associant cette fois l'action de la fortune à une topique des chœurs de Sénèque, l'exaltation de l'aurea mediocritas :

Multo ille vitam tutius exigit,

Quicunque paruis priuus in adibus,

\footnotetext{
${ }^{20}$ Nous empruntons la traduction de bacchantibus à H. Lamarque, art. cit., p. 251.

${ }^{21}$ Pharsale, 7, 593-95, trad. A. Bourgery et M. Ponchont, C.U.F., 1993, p. 72.
} 
Nullum timens, nulli timendus,

Pelle sub exigua quiescit. (Iulius Cassar, v. 236-39)

Il mènera une vie bien plus sûre,

le particulier dans son humble demeure,

lui qui ne craint personne et n'est craint de personne

mais se sent bien dans sa peau exigue $\ddot{e}^{22}$.

Cependant le schéma tragique du revers de fortune est concurrencé par le modèle de l'Hercule l'Eta de Sénèque à qui Muret fait explicitement référence par de nombreux emprunts textuels : la pièce s'ouvre ainsi sur un monologue de César qui demande à Jupiter de le recevoir parmi les astres et s'achève par l'apothéose de ce dernier. Cette fin heureuse, empruntée à Sénèque, n'a pas la préférence d'Aristote ${ }^{23}$, suivi par de nombreux théoriciens du genre, mais elle est prescrite ou tolérée par plusieurs théoriciens de la Renaissance, attentifs à concilier la crainte et la pitié avec la «surprise admirative» (admiratio $)^{24}$. Le renversement final concourt ici à préserver le caractère héroïque de César. En concluant sa tragédie sur l'apothéose de César, Muret hérite certes d'une longue tradition antique ${ }^{25}$, mais il songe peut-être à la suite de l'Énéide due à Maffeo Veggio, qui s'achève précisément par l'apothéose d'Énée décrite à l'imitation du récit par Ovide de celle de César ${ }^{26}$.

\section{Deux logiques antinomiques : César tyran ou père de la patrie?}

Muret juxtapose donc deux logiques tragiques antinomiques : César apparaît tantôt comme un héros coupable d'hybris et justement châtié par Némésis, tantôt comme la victime innocente du crime monstrueux de Brutus, alors identifié au fu-

\footnotetext{
${ }^{22}$ On aura reconnu une citation d'Horace, Satires 1, 6, 22.

${ }^{23}$ Selon Aristote, une tragédie réussie doit illustrer le passage du bonheur au malheur, plutôt que du malheur au bonheur, Poétique, 13, 53a12-17.

${ }^{24} \mathrm{~F}$. Dubois prescrit ainsi une issue heureuse pour le poème héroïque, comique ou tragique (Francisci Syluii Ambianatis Pæetica, Parisiis, in officina Iodoci Badii, 1520, B vi ${ }^{\mathrm{r}}$ ). Dans son commentaire du passage, J. Lecointe justifie ce schéma par la prédominance de la comédie, mais aussi par une conception du dénouement comme une restitution de l'unité troublée par la crise dramatique (La Poétique de François Dubois, Mémoire d'Habilitation soutenu à l'Université de Paris IV, en janvier 2000, p. 39). Plus mesuré, Scaliger rappelle cependant que de nombreuses tragédies ont une fin heureuse (Nec minus latce tragæedice non pauca.), Potices libri septem, éd. cit., 3, 96, p. 145. A. Minturno, pour sa part, prend l'exemple d'une tragédie qui mettrait en scène la mort du Christ dont la fin susciterait tout autant l'horreur et la pitié que l'admiration, Antonii Sebastiani Minturni de pœta, Venetiis, apud F. Rampazetun, 1559, p. 183-84.

${ }^{25}$ Virgile, Bucoliques, 9, 47-50 ; Ovide, Métamorphoses, 15, 804 ; Suétone, Jules César, 88.

${ }^{26}$ Comme le héros du Iulius Casar, Énée est fixé parmi les astres par sa mère et transformé en dieu indigète (v. 626-30). Parue à Pavie en octobre 1428, cette suite figure dans de nombreuses éditions de l'Énéide à la Renaissance. Le texte a été récemment édité et traduit par M. C. J. Putnam, Maffeo Vegio Short Epics, The I Tatti Renaissance Library, Harvard University press, Cambridge, 2004.
} 
rieux des tragédies de Sénèque et auteur d'un parricide sacrilège ${ }^{27}$. Or, ces logiques antinomiques sont héritées des débats antiques sur le meurtre du dictateur et en particulier de la fameuse question posée par Cicéron dans la seconde Philippique : les hommes qui ont tué César sont-ils des libérateurs ou des assassins ? (II, 30-31). Dans les Philippiques, Cicéron démontre que ce sont des vengeurs de la liberté, mais les césaricides resteront marqués de la double qualification contradictoire de tyrannicides et de parricides ${ }^{28}$.

Lorsque Brutus, à l'acte II, s'exhorte à l'action dans une longue suasoire, il justifie le meurtre par l'aspiration de César à la royauté et son désir de restaurer la liberté :

\author{
At nomen illud refugit, et oblatas sibi \\ Reicit coronas. Fingere hoc, et ludere est : \\ Nam cur Tribunos igitur amouit loco? (Iulius Casar, v. 135-137)
}

Pourtant, il fuit ce nom et les couronnes qu'on lui offre,

il les rejette. Mais ce n'est là que feinte et jeu.

Pour quel motif a-t-il donc écarté les tribuns ?

Le début du vers 135 fait allusion à une réponse donnée par César à la foule qui, à son retour des Féries Latines, le salua du nom de roi ; il aurait alors répondu « Je suis César et non pas roi »(Casarem se, non regem esse $)^{29}$. La mention des couronnes rejetées fait probablement référence à l'affaire plus connue des Lupercales, fréquemment citée par Cicéron dans les Philippiques : lors de cette cérémonie, le 15 février 44, Antoine proposa à trois reprises le diadème à César; à trois reprises, celui-ci le refusa ${ }^{30}$. Le vers 137, enfin, évoque la destitution des tribuns C. Apidius Marullus et L. Cæsatius Flavus qui avaient fait enlever une couronne placée par un jeune homme sur la statue de César ${ }^{31}$. Une fois ces trois arguments énoncés, Brutus expose le conflit tragique dont il est la proie en raison des bienfaits dont il est redevable à César. Alors que dans le De Beneficiis de Sénèque, Brutus se demande s’il

\footnotetext{
${ }^{27}$ Calpurnie qualifie les meurtriers de parricida nefandi (v. 485), tandis que le chœur les traite d'impurs (impuri, v. 511) et de sacrilèges (sacrilega manus, v. 512). Il évoque leur furor (v. 516), en appelle à la vengeance des Furies et voue les assassins aux châtiments de l'Enfer en citant les supplices réservés à Ixion ou à Sisyphe dans l'Antiquité (v. 514-528).

${ }^{28}$ Coluccio Salutati reprend le débat dans son Tractatus de Tyranno et en particulier au chapitre IV qui s'ouvre sur la formule des Philippiques, éd. F. Ercole, Berlin, Dr. Walter Rothschild, 1914, p. 42.

${ }^{29}$ Voir Suétone, Jules César, 79 ; Plutarque, César, 60 ; Appien, Guerre civile, 2, 16, 108 ; Dion Cassus, Histoire romaine, 44, 10.

${ }^{30}$ Voir Philippiques 2,$85 ; 3,12 ; 13,31$ et 41 et Nicolas de Damas, Vie de César, 21, 71-74 ; Suétone, Jules César, 79; Plutarque, César, 61 ; Appien, Guerre civile, 2, 16, 109 ; Dion Cassius, Histoire romaine, 44, 11 et S. Weinstock, Diuus Julius, Oxford, Clarendon Press, 1971, 331-40.

${ }^{31}$ Suétone, Jules César, 79 ; Plutarque, César, 74.
} 
«aurait dû accepter la vie du Divin Jules, puisqu'à ses yeux c'était un devoir de le tuer $»^{32}$, il se demande ici s'il doit rendre à César son bienfait :

At mihi et honores, et semel vitam dedit.

Plus patria illis omnibus apud me potest.

Qui se tyranno in patriam gratum exhibet,

Dum vult inepte gratus esse, ingratus est.( Iulius Casar, v. 138-141)

Pourtant il m'offrit les honneurs et une fois la vie $\mathrm{i}^{33}$

Mais sur moi la patrie peut bien plus que cela.

Rendre grâce au tyran contre les intérêts de la patrie,

c'est gratitude déplacée et donc ingratitude.

Comme le recommande Scaliger dans sa Poétique $e^{34}$, Muret utilise une sentence pour traduire l'action de Brutus en termes de classes morales manipulables et propose ainsi au spectateur une loi morale que n'annulera pas la condamnation ultérieure du meurtre. La réponse donnée par Muret au conflit entre l'amitié privée et la raison d'État est, en effet, conforme à la résolution proposée par Cicéron à ce type de conflit de devoir dans le De Officiis :

Sape enim tempore fit ut, quod turpe plerumque haberi soleat, inueniatur non esse turpe; exempli causa ponatur aliquid quod pateat latius. Quod potest maius scelus quam non modo hominem sed etiam familiarem hominem occidere? Num igitur se adstrinxit scelere si qui tyrannum occidit quamuis familiarem? Populo quidem Romano non uidetur, qui ex omnibus praclaris factis illus pulcherrimum existimat. Vicit ergo utilitas honestatem? Immo uero honestas utilitatem consecuta est.

Souvent, en effet, il arrive, du fait des circonstances, que ce qu'on a l'habitude de tenir, la plupart du temps, pour laid, se trouve ne pas l'être. Donnons pour exemple quelque chose qui soit d'une fort grande portée : peut-il y avoir plus grand crime que de tuer, non seulement un homme, mais encore un homme ami ? Est-ce que par hasard, en conséquence, celui-là s'est rendu coupable d'un crime, qui a tué un tyran,

\footnotetext{
${ }^{32}$ Disputari de M. Bruto solet, an debuerit accipere ab diuo Iulio uitam, cum occidendum eum iudicaret. De Beneficiis, 1, 20, trad. F. Préchac, C.U.F., 1972, p. 45. Sénèque indique que Brutus devait accepter la vie, toutefois sans regarder César comme son père puisque celui-ci n'avait conquis le droit de faire le bien que par la violation du droit. C'est dans ce passage qu'il condamne le meurtre, non d'un point de vue moral, mais comme une erreur de jugement historique.

${ }^{33}$ D'après Plutarque, César aurait donné l'ordre à ses officiers de ne pas tuer Brutus dans la bataille (Brutus, 5, 1). Plus loin, il indique que beaucoup ont reproché à Brutus d'avoir tué son sauveur, mais montre que son attitude envers César n'en fut que plus noble puisqu'il n'avait aucun grief privé contre lui (Brutus, 56, 4).

${ }^{34}$ Quum autem sententiarum duo sint modi, utrisque tota tragædia est fulcienda. Sunt enim quasi columna, aut pila quadam uniuersa fabrica illius. Scaliger, Pætices libri septem, éd. cit., 3, 96, p. 145A. «Il existe deux catégories de sentences et toutes deux doivent étayer toute la tragédie. Elles sont en effet comme les colonnes ou les piliers de tout cet édifice. »
} 
quoiqu'il fût son ami ? Ce n'est pas l'opinion, certes, du Peuple romain qui, parmi toutes les actions d'éclat juge celle-ci la plus belle. L'utilité l'a donc emporté sur la beauté ? Bien au contraire, c'est la beauté qui rejoignit l'utilitée ${ }^{35}$.

Suivant le modèle stoïcien de l'oikeiosis sociale, Cicéron organise la société humaine en cercles concentriques de plus en plus larges partant de l'individu, pour s'étendre au cercle de la famille, des proches, de la patrie, pour englober l'humanité entière. Cette organisation lui permet d'affirmer la prédominance absolue du lien unissant l'homme à sa patrie sur tous les autres liens ${ }^{36}$. On comprend pourquoi Cassius célèbre la ferme droiture (rigido ... honesto, v. 181-82) de Brutus.

Le discours des tyrannicides après le meurtre s'inspire aussi des lieux du discours démonstratif, intimement liés à une tradition philosophique. Pour rendre plus sensible le caractère odieux de César, les césaricides multiplient les représentations pathétiques de la servitude, dans laquelle les citoyens sont plongés, et montrent que leur dignitas est anéantie ${ }^{37}$. Comme Platon et Cicéron, ils présentent le tyran comme un être asservi à ses passions et l'invective ne retient que les pratiques les plus obscènes et les plus condamnables : non seulement César est décrit comme l'ennemi du sénat, le bourreau des innocents ou la ruine des lois, mais il est accusé de pillages et de rapines et Muret se fait l'écho des dénonciations de Catulle ou de Suétone en le dépeignant comme un débauché et un efféminé ${ }^{38}$ :

O rem pudendam! mollis et vix vir satis,

Regit Quirites Martis ortos, sanguine,

Totumque nutu pathicus orbem temperat. (v. 142-144)

Quelle honte ! Un homme efféminé - à peine un homme -

Dirige les Quirites nés du sang de Mars.

D’un signe, un débauché régit tout l'univers.

Comme Cicéron, les césaricides accumulent les termes appartenant au champ lexical de la cruauté, de la bestialité, du monstrueux (ferus animus, v. 164 ; impurus ille, v. 447 ; furore nefario, v. 447 ; rabie caca, v. 448 ; cadauer obsconum, v. 455) et on reconnaît une topique oratoire héritée de la philosophi ${ }^{39}$ : c'est cette déchéance ontologique du tyran qui légitime le tyrannicide ; dans la mesure où le tyran est exté-

\footnotetext{
${ }^{35}$ De Officiis, 3, 14, 19, trad. M. Testard, C.U.F., 1970, p. 79-80.

${ }^{36}$ Voir C. Lévy, «La monstruosité politique chez Cicéron », REL, 76, 1998.

37 Gementis patria/Pressa a tyranno, v. 103-104; En dignitatis uindicem destram tua, v. 445 ; patria oppressa, v. 455 ; Quicunque mente patriam saua premit,/ Suosque ciues subdit, ut seruos (...), v. 467-68.

${ }^{38}$ Catulle, 29 et 57 et Suétone, Jules César, 49. Le premier dénonce les amours de César et de Mamurra, le second rappelle son aventure avec Nicomède puis évoque les pamphlets qui le comparent à une femme.

${ }^{39}$ Sur cette topique, voir G. Achard, Pratique rhétorique et idéologie politique dans les discours « optimates » de Cicéron, Leiden, E.J. Brill, 1981, et l'article cité de C. Lévy.
} 
rieur au genre humain, il est en dehors des liens naturels de pietas unissant les hommes entre eux et doit être éliminét ${ }^{40}$.

En même temps, le camp de César souligne le caractère légitime du pouvoir qu'il exerce. La nourrice rappelle ainsi à l'acte III qu'il est le père de la patrie et que grâce à sa clémence il s'est acquis tous ses ennemis :

Quis tam vel audax, Casarem vt petere audeat,

Vel tam impius, petere vt velit patria patrem?

Quotquot vel error vel, voluntas pertinax,

Vel inuidia Casari inimicarat tuo,

Partim verendus perculit Martis furor,

Miranda partim Casaris clementia

Seruauit, et seruando amicos reddidit. (Iulius Casar, v. 291-297)

Qui donc aurait assez d'audace pour viser César ?

Qui donc serait assez impie pour s'attaquer au père de la patrie ?

Parmi tous ceux qui, par erreur, acharnement

ou jalousie, étaient des ennemis de ton César,

les uns furent frappés par l'affreuse fureur de Mars,

et les autres épargnés par l'admirable grâce de César

qui en les épargnant en a fait ses amis.

L'argumentation est ici empruntée au Pro Marcello ${ }^{41}$. De fait, comme Colucio Salutati dans son Traité sur le Tyran ${ }^{42}$, Muret s'appuie sur les discours parénétiques de Cicéron, pour faire de César un homme d'état modèle ${ }^{43}$. Prenons l'exemple de la tirade qui ouvre la pièce, dans laquelle à l'instar d'Hercule dans l'Hercule sur l'Eta de Sénèque, César énumère ses exploits guerriers et civils et demande à Jupiter de le recevoir au ciel :

Iam vel mihi, vel patria vixi satis :

Quid teneor vltra? Iam mihi exactum est, geri

\footnotetext{
${ }^{40}$ On retrouve cette conclusion dans l'introduction de Muret à la troisième Catilinaire : empruntant la métaphore du corps dont il est nécessaire de retrancher les parties malades, utilisée par Cicéron dans le De Officiis $(3,32)$, les Catilinaires. $(1,31)$ et les Philippiques $(8$, 15), Muret identifie les conjurés à des membres malades et loue Cicéron pour avoir soigné ce qui pouvait être soigné et pour avoir amputé les parties qui semblaient ne pouvoir être soignées (Opera Omnia, éd. D. Ruhnken, Lugduni Batavorum, apud S. et J. Luchtsmans, 1789, p. 605-6).

${ }^{41}$ Voir en particulier Pro Marcello, 7, 21 ; 6, 17 (Quos amisimus ciuis, eos Martis uis perculit, non ira uictoria.) et 6, 20.

${ }^{42}$ Après avoir défini le tyran (chapitre I) et montré qu'il est légitime de le tuer (chapitre II), Coluccio Salutati examine si César doit être compté parmi les tyrans (chapitre III). C'est Cicéron qui lui permet de répondre par la négative. Voir éd. cit., p. 34-41.

${ }^{43}$ Le Pro Marcello ou le Pro Ligario, prononcés en présence et à l'adresse de César, en 46 et en 45, font, en effet, l'éloge de la générosité de César à l'issue de la guerre civile.
} 
Sago, togáque quicquid eximium potest.

Hostes perempti, ciuibus leges dato,

Digestus annus, redditus sacris nitor,

Compostus orbis : cogitari nec queunt

Maiora cuiquam, nec minora à me geri. (Iulius Casar, v. 27-40)

Pour moi, pour ma patrie, j'ai désormais assez vécu.

Pourquoi me retenir encore ? J'ai déjà accompli

en cuirasse et en toge les plus beaux exploits.

Les ennemis anéantis, les lois données aux citoyens,

la division de l'an, l'éclat rendu aux cultes,

l'univers pacifié : nul ne saurait concevoir mieux

et je ne puis faire moins bien ${ }^{44}$.

Le dictateur semble ici répondre à Cicéron qui, dans le Pro Marcello, lui reproche de considérer sa mission comme terminée et lui explique tout ce qu'il lui reste à faire, «relever tout ce que la guerre a ruiné, tout ce que son inévitable brutalité a ébranlé et renversé ; réorganiser la justice, rétablir le crédit, réfréner les passions, favoriser la repopulation, opposer au désordre et à la déliquescence générale les liens sévères de la légalité ${ }^{45}$. Il lui enjoint donc de laisser "à la triste sagesse des philosophes le mépris de la mort » et poursuit ainsi :

Itaque illam tuam praclarissimam et sapientissimam uocem inuitus audiui : «Satis diu uel natura uixi uel gloria. » Satis, si ita uis, fortasse natura, addo etiam si placet, glorice; at, quod maximum est, patrice certe parum. Quare omitte, quaso, istam doctorum hominum in contemnenda morte prudentiam, noli nostro periculo esse sapiens. Sape enim uenit ad meas auris te idem istud nimis crebro dicere, satis te [tibi] uixisse.

Aussi ne t'ai-je entendu qu'avec douleur dire cette parole si belle de sagesse : «J'ai bien assez vécu pour la nature comme pour ma gloire!» Assez peut-être, si tu y tiens, pour la nature ; j'ajouterai même, si tu le veux, pour ta gloire ; mais, et c'est là l'essentiel, pour la patrie certainement trop peu. Laisse donc, je t'en prie, à la triste sagesse des philosophes le mépris de la mort, ne sois pas un sage au risque de notre perte. Plus d'une fois il m'est revenu que trop fréquemment tu déclares avoir bien assez vécu [pour toi] ! ${ }^{46}$

\footnotetext{
${ }^{44}$ Les «ennemis » évoqués sont probablement les Pompéiens battus à Thapsus (46) puis Munda (45); Muret fait ensuite allusion à l'ensemble des mesures politiques prises par César une fois la paix rétablie (Suétone, Jules César, 41-44); la réforme la plus célèbre est celle du calendrier (Suétone, Jules César 40 et Plutarque, César, 59), mais le dignitaire religieux que fut César sut aussi faire de la politique religieuse un des fondements de son gouvernement ouvrant la voie au futur Auguste (voir S. Weinstock, op. cit., p. 28-34).

${ }^{45}$ Pro Marcello, 8, 23, trad. Marcel Lob, C.U.F., 1968, p. 45-46.

${ }^{46}$ Pro Marcello, 8, 25, trad. cit., p. 46.
} 
Muret reprend les deux formules cicéroniennes, signal qui permet à l'auditeur érudit d'identifier la source et substitue patrice à glorica : l'énumération des mesures prises par le dictateur montre que César a totalement achevé sa mission, conformément aux injonctions de Cicéron. Il peut donc légitimement aspirer à la mort et à la sagesse des philosophes ${ }^{47}$.

\section{L'exemplarité de César}

Ce double discours de la tragédie a été mis en parallèle avec les événements contemporains : Henri Lamarque évoque prudemment les révoltes de la gabelle en Aquitaine et l'assassinat du lieutenant du roi à Bordeaux en août $1547^{48}$. La part d'ombre de Muret, emprisonné au Châtelet en 1553, pour sodomie ou pour meurtre, et brûlé en effigie à Toulouse en 1554, pour être huguenot et sodomite, incite à rappeler l'importance de la figure de Brutus dans les théories de la résistance élaborées par les protestants ${ }^{49}$. Certes, les écrits de Muret qui concernent la religion sont de stricte obédience catholique ${ }^{50}$, mais en raison de sa fréquentation de milieux huguenots et de l'influence de sa tragédie sur le César de Jacques Grévin, on peut noter, comme le fait Pierre Blanchard, que le Iulius Cassar contenait des idées qui allaient «enflammer l'opinion $»^{51}$. C'est cependant la mise en scène d'un discours monarchique qu'ont retenue les tenants d'une interprétation politique de la tragédie, suggérant que la pièce pouvait célébrer la victoire de Cérisoles en 1544, ou exalter François I ${ }^{\text {er }}$ après sa mort en $1547^{52}$. En 1552, au moment de sa publication dans le recueil des Juvenilia dont la préface célèbre Henri II, comparé à Auguste ${ }^{53}$, la tragédie répond probablement à une visée parénétique, qui associe l'éloge du souverain clément, à la fois conquérant, législateur et père de la patrie, et la condamnation d'une dérive de la monarchie vers la tyrannie.

Une autre manière de résoudre l'antinomie entre l'exaltation, puis la condamnation du meurtre de César est de lire la tragédie comme une mise en scène du conflit entre l'idéal et l'action politique: selon Manfredi Piccolomini, Muret

${ }^{47}$ C'est ainsi qu'il reprend notamment à son compte le topos stoïcien de la mort opportune exprimé au vers 44 sous la forme d'une sentence : Mors propera nunquam, sera nonnumquam venit. La fin de la tragédie met en scène la satisfaction de ses vœux puisqu'après l'assassinat, comme Hercule, César revient sur scène pour consoler sa femme et lui explique qu'il a échappé à ses assassins grâce à l'intervention de sa mère.

${ }^{48}$ Art. cit., p. 257.

${ }^{49}$ Voir sur ce point O. Jaszi et J. D. Lewis, Against the Tyrant. The tradition and theory of tyrannicide, Glencoe, Illinois, The Free press and the Falcon's garden Wing press, 1957, p. 51-52.

${ }^{50}$ C'est le cas du discours sur la dignité de le théologie, publié peu avant le Iulius Caesar : De dignitate ac prastantia studii theologici, habita Lutetice Parisiorum Non. Febr. 1552, Opera omnia, éd. Frotscher citée, t. I, p. 114-21.

${ }^{51}$ Voir éd. cit., p. 44-47.

${ }^{52}$ Voir J. Pineaux, art. cit., p. 217 et H. Lamarque, art. cit., p. 257-58.

${ }^{53}$ Quod si est, bona spes me tenet fore, vt sub HENRICO rege Christianissimo, tanquam olim sub Augusto, poëtarum ingenia excitentur, éd. cit. 1552, p. 11. «Si c'est le cas, j' ai bon espoir que sous le règne du roi très chrétien Henri, comme autrefois sous le règne d'Auguste, les talents des poètes seront stimulés. » 
exprime des conceptions proches de celles de Tacite qui tout en éprouvant un sentiment nostalgique pour les vertus de la République montra que la destinée appelait un nouvel ordre politique ${ }^{54}$. C'est cette incapacité d'un individu à changer le cours de l'histoire que semble regretter La Boëtie lorsqu'il fait l'éloge des tyrannicides tout en déplorant les conséquences funestes de leur acte puisque la liberté mourut avec eux $^{55}$.

Ce n'est pas, nous semble-t-il, la problématique de Muret et nous pensons que la condamnation de Muret s'apparente davantage à celle de Dante qu'à celle de La Boëtie. Dans la Divine Comédie, Brutus et Cassius se trouvent en compagnie de Judas dans la zone des traîtres envers leurs bienfaiteurs, tandis que César est dans les limbes, en bonne compagnie, avec Virgile et Aristote, comme il convient à une homme à qui seul le baptême a manqué pour être sauvé. Brutus et Cassius sont condamnés pour avoir porté atteinte à l'incarnation d'un pouvoir d'essence quasidivine, représenté par l'aigle de Rome qui figure au Paradis ${ }^{56}$. De même, dans la tragédie de Muret la consolation finale du chœur reprend le motif antique de l'immortalité des âmes vertueuses :

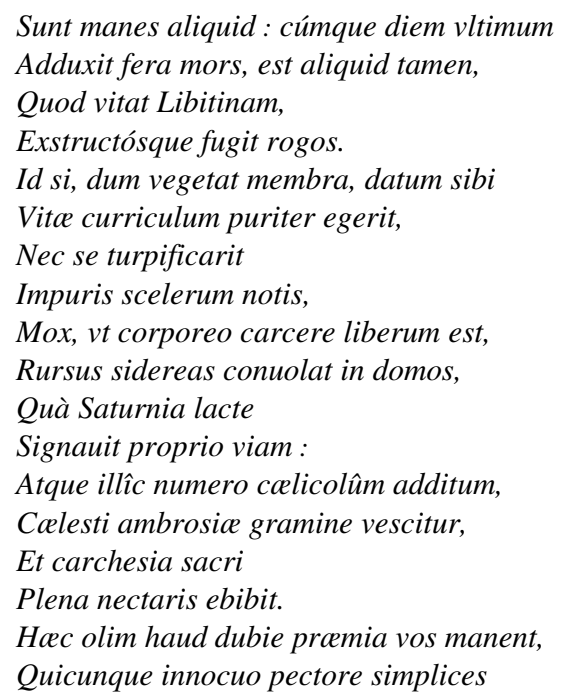

${ }^{54}$ The Brutus revival, op. cit., p. 101-102. Il accorde une grande importance à l'indécision de Brutus qui concentre tout le tragique.

${ }^{55}$ «Harmode, Aristogiton, Thrasybule, Brute le Vieus, Valere et Dion, comme ils l'ont vertueusement pensé l'executerent heureusement: en tel cas quasi jamais à bon vouloir ne defaut la fortune. Brute le jeune et Casse osterent bien heureusement la servitude, mais en ramenant la liberté ils moururent, non pas miserablement (car quel blaspheme seroit ce de dire qu'il y ait eu rien de miserable en ces gens là, ni en leur mort ni en leur vie ?), mais certes au grand dommage, perpetuel malheur, et entiere ruine de la republicque, laquelle fut, comme il semble, enterrée avec eus. », Estienne de La Boëtie, De la servitude volontaire, éd. Malcolm Smith, Droz, 1987, p. 53-54.

${ }^{56}$ Enfers, 34, 64-67 (Brutus) et 4, 123 (César). 
Virtutémque tenetis,

Et canam colitis fidem. (Iulius Casar, v. 551-570).

Les mânes ont une réalité :

quand la cruelle mort a fait paraitre le dernier jour,

il est cependant une chose qui échappe à Libitine

et fuit les bûchers élevés.

$\mathrm{Si}$, durant le temps où elle animait les membres,

elle a passé dans l'innocence l'espace de vie qui lui fut imparti,

sans se souiller des taches impures des crimes,

aussitôt qu'elle est délivrée de la prison corporelle

elle vole à nouveau vers les demeures célestes

par où la Saturnienne

a balisé la route de son propre lait,

et là, portée au nombre des habitants du ciel,

elle se nourrit de l'ambroisie céleste,

et boit de pleines coupes

de nectar sacré.

Ces récompenses vous attendent sans nul doute,

vous qui, avec simplicité et innocence,

observez la vertu

et honorez l'antique foi.

Muret concilie ici vocabulaire philosophique et représentations poétiques. La formulation des vers 551-554 est empruntée à Properce $(4,7,1-2)$ et à Horace $($ Odes 3,30 , 6-7), mais ensuite les notions philosophiques et morales dominent. L'élévation céleste de l'âme a trouvé un large écho dans l'Antiquité, que l'on pense notamment au mythe d'Er, au songe de Scipion et au commentaire de Macrobe qui met l'accent sur la vertu politique, ou au développement eschatologique d'Anchise dans l'Énéide ${ }^{57}$. De même à la fin du livre IV de la Consolation de la philosophie de Boèce, consacré à la providence divine, l'exemple d'Hercule et de son ascension au ciel est suivi d'une exhortation à suivre les nobles traces de ce grand modèle ${ }^{58}$. Or, le nectar ou l'ambroisie sont aussi une métonymie du Paradis. Dans la mesure où le chœur précédent annonce le châtiment des meurtriers en évoquant les tourments des Enfers, on pense au système de rétribution de la religion chrétienne, d'autant que dans le discours sur la «précellence de la théologie» qu'il prononça en février 1552, l'année

\footnotetext{
${ }^{57}$ Voir Platon, République, 10, 514c-621d ; Phèdre, 247b ; Cicéron, De republica, 6, 16 ; Macrobe, Songe, 1, 4, 5 et 1, 15, 1-7 ; Virgile, Énéide, 6, 713-51.

58 Vltimus calum labor inreflexo/Sustulit collo pretriumque rursus/Vltimi calum meruit laboris./Ite nunc fortes ubi celsa magni/Ducit exempli uia! (...). 4, 7, 29-33. «Car, ultime travail, il soutint le ciel sans ployer l'échine ; ce qui lui valut en retour de cet ultime travail, de mériter le ciel. Et maintenant, courage! Allez là où conduit le noble voie de ce grand exemple.», trad. J.-Y. Guillaumin, Paris, Les Belles Lettres, 2002, p. 124. Voir Sénèque, Hercule furieux, v. 69 et suivants.
} 
même de la parution des Iuuenilia, Muret décrit en des termes assez proches la rétribution des âmes après la mort ${ }^{59}$.

De fait, si Muret contamine dans la tragédie les figures classiques de la transcendance et fait intervenir à la fois les dieux de la mythologie, en particulier Jupiter et Vénus, et les figures et les divinités plus spécifiques à la tragédie comme Tychè ou Némésis, il pratique comme ses contemporains, une interprétation allégorique de la mythologie antique, qu'il justifie dans son Discours sur la précellence de la Théologie, puis dans son commentaire du De providentia où il lit Sénèque à la lumière de Jean Chrysostome ${ }^{60}$. C'est ainsi que les interprétations éthiques du revers de fortune proposées dans la tragédie s'apparentent aux justifications de la Providence stoïcochrétienne fournies dans le commentaire du De Providentia. Le malheur des puissants permet de vérifier la toute puissance de la divinité et d'empêcher l'homme de trop se fier en ses forces, mais il met aussi en valeur le courage des héros, qui, comme Brutus et César, choisissent d'affronter la mort et d'accepter leur destin. Muret rejoint par là les analyses du De fato et fortuna de Coluccio Salutati qui prend l'exemple du meurtre de César pour montrer que la providence doit être conjuguée avec le libre-arbitre ${ }^{61}$ : certes la fin de la tragédie présente Brutus comme l'instrument de la providence divine qui récompensera César, mais le meurtre est aussi le résultat de la volonté de Brutus, comme le montre la suasoire de l'acte II ; de même la mort est librement consentie et même désirée par César. C'est pour cette raison, nous semble-t-il, que Muret ne signale pas que César meurt devant la statue de Pompée. Cet élément tragique, mentionné par Nicolas de Damas, Plutarque ou Dion Cassius ne pouvait manquer de rappeler l'exemple de la statue de Mitys, cité par Aristote pour définir l'action tragique ${ }^{62}$. Or, Muret voulait précisément éviter que César n'apparaisse comme une victime de son beau-frère: la visée morale de la pièce impliquait que la mort du dictateur fût voulue par ce dernier et non subie. La célébration de la Providence divine n'annule pas la liberté de l'homme et justifie la célébration de la vertu.

Revenons donc sur les derniers vers de la tragédie, précédemment cités :

Hac olim haud dubie pramia uos manent.

Virtutemque tenetis,

Et canam colitis fidem.

\footnotetext{
${ }^{59}$ Quid porro de iisdem rebus Theologi ? Animum hominis simplicem quandam et individuam esse naturam, ad exemplar divinitatis expressam, licet ortus participem, tamen interitus expertem, quæ corpore soluta, si se pure ac pie gesserit, in colum evolet, æternam illic Dei beneficio adeptura felicitatem; sin se malis iisque inexpiabilibus cupiditatibus corrumpendam dederit, ad inferos nunquam desituris damnanda suppliciis deprimatur. De dignitate ac prostantia studii theologici, éd. Frotscher citée, t. I, p. 119.

${ }^{60}$ Cum Seneca librum De Prouidentia interpretaturus esset. Oratio III. Habita Roma III. Non. Iun. MDLXXV, éd. Frotscher citée, t. I, p. 311-20.

${ }^{61}$ C. Salutati, De fato et fortuna, 2, 8, éd. C. Bianca, Firenze, Leo S. Olschki Editore, 1985, p. 67-71.

${ }_{62}$ Voir Aristote, Poétique, 9, 1452a1-11; Nicolas de Damas, Vie de César, 23, 82-83; Plutarque, Brutus, 66 et Dion Cassius, Histoire romaine, 44, 52.
} 
L'expression canam... fidem est utilisée par Virgile dans l'Énéide $(1,292)$ où elle désigne l'antique Bonne Foi aux cheveux blancs, à laquelle Numa Pompilius bâtit un temple sur le Palatin. En reprenant la formule, Muret unit, comme Virgile, l'amour de la patrie et le désir de gloire aux vertus civilisatrices instaurées par Numa, mais rappelle aussi la loyauté que le roi et ses sujets se doivent. Il ne faut pas négliger l'acception religieuse du terme fides. De fait, Muret contamine deux sources dramatiques, l'Hercule l'Eta de Sénèque, qui s'achève sur la recommandation d'affronter l'adversité avec courage: Numquam Stygias fertur ad umbras/inclita uirtus : uiuite fortes, v. 1983-84, mais aussi le chœur final de l'Ecérinide de Mussato, imité de celui de l'Hercule l'CEta. Or, Mussato qui lit les tragédies de Sénèque à la lumière de la pensée de Boèce, substitue à la catastrophe tragique l'exaltation de la justice divine ${ }^{63}$. Le chœur final, imitant la structure grammaticale des vers de Sénèque, engage à vivre dans la foi (Hoc perpetuo durat in euo/regula iuris. Fidite iusti, v. 617-18) et célèbre dieu qui récompense les justes et condamne les coupables (v. 624). Chez Mussato, leçons politique et religieuse se complètent puisqu'il s'agit de célébrer la défaite du gibelin, Ezzelino da Romano dans la bataille qui l'oppose aux troupes de l'archevêque de Ravenne. L'ordre divin se substitue à celui que la violence avait établi.

Chez Muret la contamination des deux sources et la célébration conjointe de virtus et de fides constituent peut-être une référence, discrète certes, à la question théologique qui occupe tous les esprits pendant ces années, c'est-à-dire la question de la grâce divine qui opposa Luther à Erasme. Comme dans son discours sur la précellence de la théologie, Muret exprimerait ici la voie médiane qui sera adoptée par le Concile de Trente ${ }^{64}$ en rappelant l'importance conjointe de la foi et des œuvres. Par cette pointe finale, il répondrait aux tragédies protestantes contemporaines de Buchanan ou de Théodore de Bèze.

Pour conclure, si Muret n'est pas Shakespeare, le Iulius Caesar remplit incontestablement sa mission : il contribue à la renaissance du genre de la tragédie «prétexte », il est école d'éloquence et il fournit un support privilégié pour l'étude de l'histoire ancienne, tout en proposant une réflexion d'actualité sur l'éthique du pouvoir et sur les débats théologiques contemporains. Alors qu'Horace prescrit aux auteurs dramatiques de veiller à la cohérence des caractères ${ }^{65}$, Muret sacrifie la cohérence du personnage de César et en accentue l'ambivalence afin de concilier visée cathartique et observation des vices et des vertus. La multiplication des schémas tragiques, plus encore que le conflit des légitimités auquel nous a habitués la tragédie antique, peut surprendre le lecteur moderne, mais d'une part la coexistence de points de vue contradictoires est caractéristique de la Renaissance et surtout l'opposition de chacun des héros à son double négatif a ici paradoxalement pour effet de réduire l'opposition traditionnelle entre Brutus et César et l'antithèse entre vertu et grandeur, pureté d'âme et fortune. Tandis que le constat de la toute puissance de la Fortune et du hasard incite Machiavel à définir dans le Prince un art de

\footnotetext{
${ }^{63}$ Voir l'introduction de J.-F. Chevalier, Ecérinide (...), Paris, Les Belles Lettres, Classiques de l'Humanisme, 2000, p. LXXIX-LXXXIX.

${ }^{64}$ Le décret de la justification a été voté en janvier 1547.

${ }^{65}$ Art poétique, 119-127.
} 
gouverner efficace sans préoccupation d'ordre moral. Muret se fonde sur une eschatologie pour justifier une éthique et une mystique du pouvoir et subordonner toute action politique à la poursuite d'une fin juste. Ainsi, il ne conclut pas sur la forme du régime, mais sur la vertu des dirigeants. César incarne ainsi un idéal héroïque qui associe la magnanimité guerrière, les vertus civilisatrices célébrées par la propagande augustéenne et la grandeur d'âme des philosophes. Plus généralement, la tragédie célèbre un idéal éthique qui concilie la grandeur stoïcienne et l'humilité chrétienne.

V. Leroux

Université de Reims. 